era when the APS processes compuscript submissions, expects to soon have a preprint server (so that authors can benefit from nonanonymous comments before more traditional anonymous refereeing) and is "positioning" to deliver all its research journals online (PRL has been online since July 1995 - with a WWW interface since August 1995 - and the routine conversion of $100 \%$ of MS to electronic formats is in sight).

\section{Hypertext Potential to be Exploited}

Billed as a "fully electronic", peer-reviewed journal in spite of the availability of the traditional paper format, Elsevier Science (http://www.elsevier.nl/) is to launch in early1996 New Astronomy covering all fields of astronomy and astrophysics. It will be made available via WWW and over local area networks (LAN). It is claimed that the WWW edition will represent a new format for publishing research and letter articles through the use of hypertext links to bibliographic databases, scientific data sets, tabular materials, and sets of (colour) figures, animation or sound (which can be part of a submitted article or located elsewhere on WWW). Other features include a short publication time, at least for publication on WWW (one month for the refereeing process; one month between acceptance and WWW release) and free subscriptions (covering the WWW version together with the LAN and printed editions) during the start-up period of at least 1996.

\section{Program Library Integrated}

The Computer Physics Communications Internal Program Library which holds some 1500 programs, with about 150 new programs added annually, (http://www.cs.qub.ac.uk/cpc/; cpc@qub.ac.uk) was established in 1969 at the Queen's University of Belfast under a UK government grant as a self-supporting repository for the storage and dissemination of refereed computer programs for non-profit use, descriptions of which were published in Computer Physics Communications (CPC). As from January 1996, the Library has become an integral part of the journal so that a subscription to CPC includes access to the library (users in subscribing institutions can register directly).

\section{IOPP Journals Go Online}

The IOPP (http://www.iop.org/) now has 14 journals on WWW and the remaining 17 will follow within a few weeks. Any researcher in a subscribing institution, once registered, will be able to access via WWW papers up to three weeks before paper publication (initially as HTML files for headers and interface, Adobe Acrobat PDF and PostScript files for full text, maths and graphics, and $\mathrm{T}_{\mathrm{E}} \mathrm{X}$ for some journals).

Physics Online Library Launched Springer has set up the Physics Online Library $(P O L)$ - a digital collection of original peer-reviewed contributions coming from a variety of sources, as well as electronic articles in all areas of physics. $P O L$ will incorporate online versions of Zeitschrift für Physik A, B, C, and $D$ to be released in the course of 1996 .

This comes at a time when Springer has announced that a Helpdesk on Internet will be

\title{
Archiving On-line Journals
}

A number of purely electronic (without printed versions), peer-reviewed journals in the physical sciences or related areas are now being offered, generally free of charge to anyone with WWW access. Claims include: extremely rapid publication; instant world-wide distribution: increased readership (since often no subscription fees); immediate downloading and printing of articles; a level of service far beyond the capabilities of paper journals (video and audio capabilities; viewing of digitized items, e.g., full-colour photographs); rapid communicate with authors through direct email links within the text; the posting of comments; debate and discussion groups moderated by an expert; thematic searching. However, most journals do not discuss archiving: some claim "universal access to their results in perpetuity" but do not explain how this is done. Only occasionally is a specific archiving procedure mentioned, as detailed in the table below giving: Title. Start of publication/structure/fees/access/publisher Archiving procedure

Complexity International April 1994/continuous addition/no fees/WWW/Australian National Univ. http://life.anu.edu.au/ci/ci.html

Electronic J. of Combinatorics Nov. 1994/3 issues/no fees/WWW/Georgia Tech in cooperation with the American Mathematical Soc. - http://ejc.math.gatech.edu:8080/Journal/journalhome.html

Internet J. of Nitride Semiconductor Research Jan. 1996/no fees for readers; page charges/WWW/under the auspices of the Materials Research Soc. - http://nsr.mij.mrs.org/

J. of Molecular Modelling March 1995/continuous addition/fees for institutions \& individuals/FTP ; WWW abs. of accepted papers/Univ. Erlangen-Erlangen in cooperation with Springer - http://derioc1.organik.uni-erlangen.de/ info/JMOLMOD/jmolinfo.html

2 year min. FTP availablity; CD-ROM issued

J. of Universal Computer Science Nov. 1994/12 issues/none in '95-96/WWW; some features only with Hyper-G viewers/Univ. of Graz in Collab. with Springer - http://hgiicm.tu-graz.ac.at/CJUCS_root

CD-ROM issued

Mathematical Physics Electronic Journal (March 1995/1 issue/no fees/ WWW; PostScript files, wih logo, volume, paper number, \& time stamps/University of Texas - http://www.ma.utexas.edu/mpej/MPEJ.html

Files maintained by Univ. of Texas; printed copy of each volume sent to US Library of Congress

Molecular Vision Oct. 1995/1 issue/no fees/WWW/Emory Univ., USA http://www.cc.emory.edu/MOLECULAR_VISION?index.html

Theory \& Applications of Categories July-1995/continuous addition/individuals free; institutional subscription/WWW/ Int. Steering Committee- $h$ ttp://www.tac.mta.ca/tac/ Electronic archives maintained by

available for subscribers to the 110-year old Landolt-Bernstein data collection, together with electronic tools and complete electronic versions to improve retrieval and document delivery procedures.

\section{Few-Body Systems Electronic}

The electronic version of Springer's FewBody Systems (http://fbs.kfunigraz.ac.at/Cfbs/) has finished a one-year test phase. A subscription (either individual with password protection or institutional with machine protection) to the printed plus electronic versions is now needed to access all its features. The clients Harmony (for UNIX) and Amadeus (for MS Windows) are needed to fully exploit the Graz University Hyper-G hypermedia system which the journal uses. Access via World-Wide Web or Gopher is possible, but some advanced features of Hyper-G (e.g., following links in PostScript documents) are not supported.

\section{Will SGML Make a Comeback ?}

Everyone who publishes WWW pages must have a working knowledge of the Hyper-Text Markup Language (HTML) which allows text to embed simple formatting information and links. But HTML is a moving target: HTML 2.0 is an Internet Draft and many issues (tables, math, character sets, etc.) do not form part of it, and HTML 3.0 is in flux. HTML is one instance of the infinite family of Standardised Generalised Markup Language (SGML) metalanguages used for defining markup. SGML is useful for archiving text in databases and for reproduction on different media. With the notable exception of the American Physical Society, publishers have tended to adopt in-house versions of SGML.

The widespread use of HTML demonstrates that SGML could be useful for networked information. One route is to treat SGML like a specialized data format, and to launch specialized or general-purpose SGML browsers to display data. The other (better) route is to integrate SGML awareness beyond HTML into browsers. Either way, display of an SGML document could be controlled by a style sheet to define new logical tags and their default visual interpretation, using a small number of display primitives ("bold", "line break", etc.) to specify the rendition of each element type. For "wellknown" Document Type Definitions (DTDs) like HTML, style sheets could be distributed with the browser, or built in. For other DTDs, the browser could fetch a style sheet from the server. A common (or meta) style-sheet language for browsing and forms, at least, must be agreed on to take serious advantage of SGML. The Document Style Semantics and Specification Language (DSSSL) has been proposed as a general set of transformation rules, with DSSSL Lite and FOSI being used for document display. A simpler approach would to reach agreement on a minimal SGML document that is sent by a server. The browser can then do a minimal amount of parsing provided a few additional rules are agreed on. A more radical approach is to use programming languages such as Java which allow relatively difficult to develop applications, downloaded while browsing, to format text and formulae. 